\title{
Evaluation of four screening protocols for detection of extended-spectrum $\beta$-lactamase-producing members of the Enterobacteriaceae
}

\section{Gram-negative bacteria expressing} extended-spectrum $\beta$-lactamases (ESBLs) have emerged worldwide. In hospitals with a low ESBL prevalence, screening for ESBL carriage at admission may be useful in populations at risk for ESBL carriage, such as those from a facility with an ESBL outbreak or those from a geographical area with high ESBL prevalence. However, without accurate laboratory screening protocols, ESBL colonization may remain undetected, thereby favouring ESBL transmission.

In this study, we evaluated four protocols for detection of ESBL-producing members of the Enterobacteriaceae from rectal swabs and faecal samples. The aim of this study was to determine whether a preenrichment step prior to inoculation on a selective ESBL agar augments the sensitivity of the screening protocol.

Rectal swabs obtained for surveillance purposes from intensive care unit (ICU) patients at two teaching hospitals (Rode Kruis Ziekenhuis, Beverwijk, and Kennemer Gasthuis, Haarlem, The Netherlands) from January to May 2009 were included in this study. In addition, random faecal samples obtained from general practitioner (GP) patients and rehabilitation centre (RC) residents (Center for Rehabilitation, Heliomare, Wijk aan Zee, the Netherlands) were tested. Heliomare supports clients with a physical disability, a traumatic brain injury or autism. A variety of services are offered, including medical rehabilitation, vocational rehabilitation, special education, housing and sports. Specimens were obtained with a swab and suspended in $1 \mathrm{ml}$ of a liquid Amies medium (ESwab; Copan). A $10 \mu$ l suspension was inoculated four times in a single session onto/into four culture media: directly on a chromogenic agar (method A: ESBL-ID; bioMérieux); into trypticase soy broth (TSB) supplemented with $1 \mathrm{mg}$ ceftazidime $1^{-1}$ (method B); into TSB supplemented with $1 \mathrm{mg}$ cefotaxime $1^{-1}$ (method C); and into non-selective TSB (method D; without antibiotics). After incubation for $24 \mathrm{~h}$ at $36{ }^{\circ} \mathrm{C}, 10 \mu \mathrm{l}$ of the enrichment broth (methods B, C and D) was plated on an ESBL-ID plate as in method A. All plates were incubated for $48 \mathrm{~h}$ at $36{ }^{\circ} \mathrm{C}$. Species identification and determination of MICs was performed using Vitek 2 for all oxidase-negative colonies presenting different morphological aspects and growing on ESBL-ID after 18-24 h. Confirmation of ESBL production was performed using combination discs with cefotaxime, ceftazidime and cefepime with and without clavulanic acid, for isolates suspected of ESBL production according to the Vitek 2. The ESBL genes of phenotypic ESBL-positive strains were characterized using PCR and sequencing of TEM, SHV and CTX-M $\beta$-lactamase genes.

A total of 514 samples obtained from 384 patients were tested (ICU patients: 202 samples, 72 patients; GP patients: 218 samples, 218 patients; RC patients: 94 samples, 94 patients). The sensitivity calculation was based on the total number of ESBL-positive strains isolated. A total of 23 samples obtained from 21 patients tested positive for ESBL-producing members of the Enterobacteriaceae, giving an overall prevalence of $5.5 \%$. The relative prevalence of ESBLs for the different groups tested is shown in Table 1. ICU patients were more likely to be colonized than GP or RC patients. The majority of ESBL-positive samples were positive for Escherichia coli: 21 E. coli, 1 Klebsiella pneumoniae and 1 Enterobacter cloacae were isolated. The predominant ESBL gene was CTX-M (70\% of isolates, Table 2). The sensitivity of methods A, B, C and D on a sample level was estimated as $70 \%$ (16/23; $95 \%$ CI, 49-85\%), $82 \%$ (19/23; $95 \%$ CI, 62-94\%), $82 \%(19 / 23$; $95 \%$ CI, $62-94 \%)$ and $74 \%(17 / 23 ; 95 \%$ CI, 62$94 \%$ ), respectively. On a patient level, the sensitivity of methods A, B, C and D was estimated as $71 \%(15 / 21 ; 95 \%$ CI, 50$86 \%), 86 \%$ (18/21; $95 \%$ CI, 65-96\%), $81 \%(17 / 21 ; 95 \%$ CI, 59-93\%) and $76 \%$ (16/21; $95 \%$ CI, 62-94\%), respectively. With a combination of methods B and C, the sensitivity was estimated as $96 \%$ (22/ 23; $95 \%$ CI, 77-100\%) on a sample level and $95 \%(20 / 21 ; 95 \%$ CI, 76-100\%) on a patient level. Sensitivities were compared using the McNemar test for paired samples and Fisher's exact test.

In this study, a pre-enrichment step prior to direct inoculation of culture swabs onto a selective agar resulted in an increase of the sensitivity of the ESBL detection method, although these differences were not statistically significant. Only a combination of methods B and C yielded a statistically

Table 1. Prevalence of ESBL-producing members of the Enterobacteriaceae in different patient populations on a sample level and patient level

\begin{tabular}{|lcclcc|}
\hline \multirow{2}{*}{ Population } & \multicolumn{2}{c}{ Samples } & & \multicolumn{2}{c|}{ Patients } \\
\cline { 2 - 3 } \cline { 6 - 7 } & Positive (\%) & $\mathbf{9 5 \%}$ CI & & Positive (\%) & 95\% CI \\
\hline ICU & 6.4 & $3.7-10.8$ & & 15.3 & $8.6-25.6$ \\
GP & 2.8 & $1.1-6.1$ & & 2.8 & $1.1-6.1$ \\
RC & 4.3 & $1.4-10.9$ & & 4.3 & $1.4-10.9$ \\
\hline
\end{tabular}


Table 2. Prevalence of ESBL genes found

\begin{tabular}{|c|c|c|c|}
\hline & \multicolumn{3}{|c|}{ ESBL gene } \\
\hline & TEM & SHV & CTX-M \\
\hline & Escherichia coli (5) & Escherichia coli (1) & Escherichia coli (15) \\
\hline & Enterobacter cloacae $(1)^{*}$ & Enterobacter cloacae $(1)^{*}$ & Klebsiella pneumoniae (1) \\
\hline Total: & 6 & 2 & 16 \\
\hline
\end{tabular}

${ }^{\star}$ These represent the same Enterobacter cloacae isolate. It contains both a TEM and an SHV gene.

significant difference in sensitivity. Other authors concluded that a simple overnight pre-enrichment step improves the sensitivity of detection of ESBLpositive strains, and permits earlier recognition and isolation of patients who carry these strains (Murk et al., 2009).

Our data indicate that direct inoculation on a selective chromogenic medium is a simple, rapid and acceptably accurate method for performing active surveillance for ESBLs in a variety of health-care populations. Larger studies that compare different screening protocols are required to confirm these findings.
Bram Diederen, ${ }^{1}$ Chisan Chang, ${ }^{1}$ Sjoerd Euser ${ }^{1}$ and James Cohen Stuart ${ }^{2}$

${ }^{1}$ Regional Laboratory of Public Health, Haarlem, The Netherlands

${ }^{2}$ Department of Medical Microbiology, University Medical Center Utrecht, Utrecht, The Netherlands

Correspondence: Bram Diederen (bramdiederen@gmail.com)

Murk, J. L., Heddema, E. R., Hess, D. L., Bogaards, J. A., Vandenbroucke-Grauls, C. M. \& Debets-Ossenkopp, Y. J. (2009). Enrichment broth improved detection of extended-spectrum-beta-lactamase-producing bacteria in throat and rectal surveillance cultures of samples from patients in intensive care units. J Clin Microbiol 47, 1885-1887. 